10 minutes after extubation. Attenuation of airway response and Extubation quality was rated using extubation quality scale. Adverse effects, Extubation and Emergence time were recorded. Results: All data measured and analyzed using standard statistical tests (student's t-test, Chi-square, Fisher's Exact Probability tests, Man- Whitney, Wilcoxon rank test). A ' $P$ ' value of $\leq 0.05$ was taken as significant. The systolic, diastolic blood pressure and heart rate were higher in group $\mathrm{C}$ than in group $\mathrm{D}$. The need for rescue medications (nitroglycerine for hypertension, esmolol for tachycardia) was higher in group C. Extubation quality scores were superior in group D than group C. Extubation time and Emergence time were comparable in both the groups. Conclusion: Dexmedetomidine in a single dose of $0.25 \mathrm{mcg} / \mathrm{kg}$ is safe and efficacious in attenuation of hemodynamic responses and airway reflexes during extubation following spine surgery without prolonging emergence and extubation times.

\section{Effect of tranexamic acid on blood loss and quality of surgical field in trans-sphenoidal surgery}

\section{Amlan Swain, Jyotsna Wig, Nidhi Bidyut Panda, Ritesh Lamsal, K. K. Mukherjee ${ }^{1}$ Department of Anesthesiology and ${ }^{1}$ Neurosurgery PGIMER,
Chandigarh, India}

Background: The aim of this study is to assess the effect of preoperative bolus followed by intraoperative infusion of tranexamic acid on intraoperative blood loss and the quality of surgical field in trans-sphenoidal surgery. Materials and Methods: A prospective randomized double blind study was carried out in 50 ASA grade I-II patients presenting for elective trans-sphenoidal pituitary surgery for pituitary tumors. One group received bolus intravenous dose of tranexamic acid $(25 \mathrm{mg} / \mathrm{kg})$ followed by infusion $(1 / \mathrm{mg} / \mathrm{kg} / \mathrm{hr})$ of the same till the end of surgery while the placebo group received normal saline in the same amount. All patients were analyzed for preoperative color perception, intraoperative hemodynamic monitoring, hematocrit, arterial blood gas values, quality of surgical field (Boezaart scale), amount of blood loss and anaesthetic requirements. Results: The demographic, hemodynamic and anesthetic parameters, duration of surgery, amount of intraoperative fluid and urine output were comparable in both the groups. The tranexamic group had significantly better quality of surgical field (Boezaart score $2.64 \pm 0.638$ vs. $3.08 \pm 0.759$, $P=0.031)$ and lesser blood loss (334 \pm 101 vs. $495 \pm 226 \mathrm{ml}$, $P=0.02)$ than the placebo group. Conclusions: Despite widespread use in other surgical specialities, tranexamic acid is rarely used in neurosurgical operations. In standard doses, it is found to be effective in traumatic brain injury and spinal surgery. Therefore, unless contraindications exist, tranexamic acid can be tried in neurosurgery. Conclusion: Intraoperative use of tranexamic acid improves the quality of surgical field as well as reduces bleeding in patients undergoing trans-sphenoidal resection of pituitary tumors.

\section{Effect of dexmedetomidine as anaesthetic adjuvant in patients undergoing trans-sphenoidal pituitary surgery}

\author{
Renu Bala, Arvind Chaturvedi, Mihir P. Pandia, \\ Parmod K. Bithal \\ Department of Neuroanaesthesiology, AIIMS, New Delhi, \\ India
}

Background: We conducted a prospective, randomized, double-blind, placebo-control study to evaluate use of dexmedetomidine as anaesthetic adjuvant in patients undergoing trans-sphenoidal pituitary surgery. We tried to find its effect in attenuating hemodynamic response to nasal speculum insertion and on early postoperative recovery profile and intra operative consumption of anaesthetic agents. Materials and Methods: After obtaining approval from ethics committee, we enrolled 60 adult patients of either sex belonging to ASA-status I and II planned to undergo elective trans-sphenoidal pituitary surgery. Routine investigations and preoperative assessment was done as a standard protocol and injection glycopyrrolate $0.2 \mathrm{mg}$ intramuscularly was administered $30 \mathrm{~min}$ prior to surgery. In group D $(n=30)$ dexmedetomidine $1 \mathrm{mcg} / \mathrm{kg}$ over 10 minutes followed by $0.5 \mathrm{mcg} / \mathrm{kg} / \mathrm{hr}$ was given in infusion while in group C $(n=30)$ normal saline ran in the same rate. Induction of anesthesia was achieved with sleep dose of propofol, fentanyl $2 \mathrm{mcg} / \mathrm{kg}$ and rocuronium $1 \mathrm{mg} / \mathrm{kg}$. Monitoring for heart rate, ECG, non-invasive and invasive blood pressure, oxygen saturation, end-tidal carbon di-oxide, BIS, along with neuromuscular monitoring was carried out. Peri-operative hemodynamic parameters were recorded and any fluctuation (increase or decrease) were treated as per protocol. The total dose of anesthetics, analgesics, muscle relaxant, and other drugs (anti-hypertensive's) used were noted. The duration of anesthesia, surgery, nasal speculum duration and intraoperative fluids, blood loss and urine output were also recorded. Any adverse event or complication if occurred was noted. After conclusion of surgery, neuromuscular blockade was reversed and recovery profile (emergence time, extubation, time for visual examination, time for first analgesic, modified aldrete score, VAS score, Ramsay sedation score, postoperative nausea and vomiting and shivering) were recorded. The data was compiled and analyzed using SPSS for Windows version 20. Results: Our study demonstrated that patient in dexmedetomidine group had significant reduction in hemodynamic response to nasal 